\title{
HEMOSTASIS IN ERYSIPELAS - MODERN CONCEPTS
}

\author{
Tsveta Kalinova, Yoanna Velevska, Sonya Márina \\ Department of Infectious Diseases, Parasitology and Dermatovenerology, \\ Faculty of Medicine, Medical University of Varna
}

\begin{abstract}
INTRODUCTION: Erysipelas is a common, ubiquitous bacterial infection of the skin mainly caused by Streptococcus pyogenes - group A beta-hemolytic streptococcus. The current epidemiology of the disease demonstrates an upward trend in cases with primary and recurrent erysipelas, a change in the site of predilection - from face to lower limbs, and an increase of severe cases with a hemorrhagic or bulloushemorrhagic component. To date, the alterations in hemostasis due to streptococcal bacterial infection are still not well defined. Despite the advanced treatment of erysipelas, in approximately $10 \%$ of patients with hemorrhagic and bullous-hemorrhagic erysipelas, a skin necrosis has occurred. The combination of severe types of erysipelas with local and general predisposing factors such as diabetes mellitus, obesity, chronic vessel insufficiency results in recurrences and complications with high percentage of disability. In addition, streptococcal infections may have fulminant progression as for every 1 hour delay of adequate treatment, the percent of mortality increases with $7.5 \%$.

AIM: The purpose of this paper is to establish the current concepts and observations of hemostatic changes in erysipelas.

MATERIALS AND METHODS: Literature referred in MEDLINE, Web of Science, Scopus has been studied.

RESULTS: The hemostasis changes in erysipelas are not well researched. The available literature is insufficient as the studies are based on a small number of patients, which prevents any significant conclusions.

CONCLUSION: The increasing incidence of patients with severe form of erysipelas stresses the need to intensify the study of hemostasis in erysipelas. Knowing the mechanisms of coagulation changes might help to predict and prevent severe, irreversible complications.
\end{abstract}

Keywords: hemostasis, coagulation, erysipelas

Address for correspondence:

Tsveta Kalinova

Faculty of Medicine

Medical University of Varna

55 Marin Drinov St

9002 Varna

e-mail:immunefragment@gmail.com

Received: July 27, 2019

Accepted: September 12, 2019

\section{INTRODUCTION}

Erysipelas is a common, acute or chronic (recurrent) infection of the skin affecting the superficial dermis and hypodermis, lymph vessels, and peripheral lymph nodes. The disease is mainly caused by Streptococcus pyogenes, rarely by other etiological agents such as St. aureus, Str. pneumoniae, Kl. pneumoniae, E. coli (1-3).

Group A beta-hemolytic streptococcus is an obligatory human pathogen. It comprises a hyaluronic capsule, a superficial protein layer, a middle 
polysaccharidic layer, and an inner peptidoglycan layer. The basic factor of virulence - the so-called Mprotein - is able to inhibit the microbial phagocytosis $(4,5)$. Similarly, polysaccharide A has a toxic effect on cells. Peptidoglycans are cardiotoxic, pyrogenic and have a thrombolytic effect on human tissues. What is more, $S$. pyogenes has a huge number of enzymes among which are erythrogenic toxin, $\mathrm{O}-/ \mathrm{S}$-streptolysins, streptokinase, proteinase, esterase, amylase, hemolysin, CAMP-factor, DNA-ase, hyaluronidase, complement inhibitors, cysteine protease, endo- $\beta$ $\mathrm{N}$-acetylglucozaminidase, superoxide dismutase and immunoglobulin-destructing enzymes (6-10).

During erysipelas, structural and functional components of the causative agent get into interaction with the host resulting in an alteration of some coagulation indices. The reactivity of the hemostatic system is explained by the common origin of the hemostatic and immune system and has the aim to limit the spreading of an infectious agent and to help its elimination. As a response to a given infectious agent, the immune system generates an immune response, mediated by procoagulant substances. The coagulation system provides the immune system with a positive feedback by using substances with pro- and anti-inflammatory effect. This well-established balance helps to limit pathogen dissemination $(11,12)$.

$S$. pyogenes is an obligatory pathogen for the human kind, which has evolved along with the human immune system. S. pyogenes is able to activate the coagulation system and then to avoid its defense mechanisms by promoting fibrinolysis (Fig. 1). During streptococcal infection, $S$. pyogenes stimulates platelet aggregation, $\mathrm{M}$-protein-induced coupling of

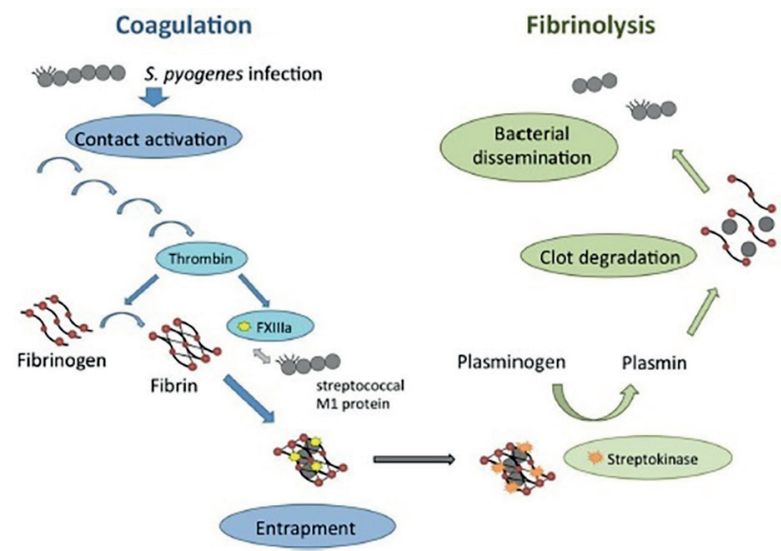

Fig. 1. Schematic representation of the interaction between Str. pyogenes and the host plasma fibrinogen and GPIIb/IIIa platelet receptors and tissue factor expression. Tissue factor is the most powerful molecule affecting coagulation cascade (13). The aim of coagulation involvement during inflammatory response is fibrin clotting and $S$. pyogenes entrapment. Nevertheless, $S$. pyogenes has developed an evolutionary strategy to escape hemostatic system defense by producing fibrinolytic enzyme streptokinase (14). Once started, the fibrinolysis enables pathogen invasion and dissemination (15).

\section{The Physiology of Coagulation}

Hemostasis works through a dynamic balance between procoagulant and anticoagulant mechanisms of the body. It consists of three main parts, structurally and functionally connected - a vascular wall, plasma systems - coagulation, fibrinolysis, kallikrein-kinin and complement - and cellular elements - mainly platelets (16-18). In 1960, Davie, Ratnoff and Macfarlane presented a schematic model of the coagulation system. They postulated the so-called "cascade" theory, and according to it there are two pathways of hemostasis activation - intrinsic pathway of coagulation (contact system) and extrinsic pathway of coagulation (tissue factor - dependent) $(15,19)$ (Fig. 2). This is an illustrative model and is only valid in vitro (16). Hemostasis develops in three phases and is subdivided into primary and secondary hemostasis. The pathological alterations may affect every phase, part or division of the hemostasis $(20,21)$.

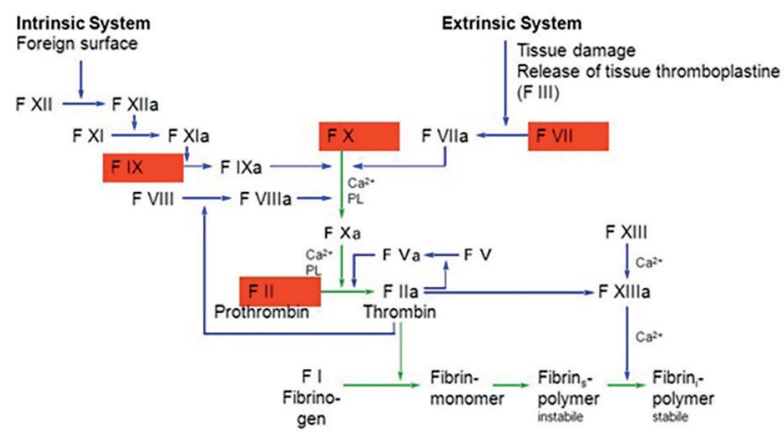

Fig. 2. A schematic model of intrinsic and extrinsic pathway of hemostasis activation

Pathophysiology of Coagulation in Erysipelas Coagulation has three phases (15):

1. Alteration in the first phase of hemostasis hypercoagulation, acoagulability, hemorrhagic syndrome 
The primary phase of hemostasis is related to the formation of an FX complex, responsible for the activation of prothrombin (prothrombinase). It is reported that during an infectious disease, an increased thromboplastin activity is observed, thus putting the patients at risk of disseminated intravascular coagulation (DIC). Bacterial streptococcal toxins are able to induce sudden activation of the coagulation leading to increased level of thrombin due to the high fibrinogen and following intravascular coagulation on macro- and microlevel. The depletion of blood clotting factors and the overwhelming fibrinolysis result in exhaustion of all hemostatic protective mechanisms and development of acoagulability, hiding the risk of hemorrhagic syndromes $(20,22)$.

\section{Alteration in the second phase of hemostasis} - coagulopathy

During the second phase of hemostasis, the nonactive prothrombin is converted into active thrombin through FXa, FVa, Ca and platelets FIIIa. Consequently, the syndrome of coagulopathy is developed $(15,20)$. Bacterial toxins play a key role in the pathophysiology of the second phase, because they are responsible for the toxic damage of liver. Alteration of the synthetic and metabolic liver function induces vitamin $\mathrm{K}$ deficiency, low level of prothrombin, F VII, IX, X, reduced activity of proaccelerin. In infections running with toxic infectious syndrome, the synthesis of all plasma factors is compromised. The diminished stability of fibrin-stabilizing factor leads to reduced resistance of the blood clot and it becomes easily destroyable $(20,22)$. Corresponding$l y$, the inadequate function of the liver is a reason for abnormal fibrinogen synthesis and its level is highly reduced. Replenishment of the fibrinogen level is realized by its extraction from the profibrin layer of the vascular wall. Consequently, the remodeled structure of the blood vessels leads to increased vascular permeability and leakage and an increase in hemorrhagic manifestations (20).

\section{Alteration in the third phase of coagulation - low levels of fibrinogen}

The third phase of hemostasis is engaged in catalytic transformation of fibrinogen into fibrin peptides and monomers. These monomers have the ability to polymerize to a fibrin clot, which later converts into stable fibrin with the assistance of FXIII.
The pathological changes observed during the third phase of hemostasis are parenchymal damage of the liver and reduction of the level of fibrinogen (23).

Changes in Hemostasis Affecting Structural and Functional Components

Streptococcal toxins are able to induce bone marrow toxicity or an autoimmune process. In 2016, E. Fokina observed increased erythrocyte deformability and decreased elasticity (24). Sjöbring et al. (2002) are pioneers in the elicitation of the streptococci-induced hemostatic changes. They proposed that M-protein of the cocci bonded with the plasma fibrinogen. Then, the formed complex adheres to the GPIIb/IIIa fibrinogen receptors of platelets (25). Oehmcke et al. (2012) showed that the soluble $\mathrm{M}$-protein was able to induce the release of procoagulant particles from the monocytes - a mechanism activating both pathways of coagulation (26). Renné (2012) noticed that the induction of contact system of coagulation resulted in the release of proinflammatory peptides - kinins. Kinins are molecules able to induce anti-inflammatory reaction against streptococci (27). Due to the pathogenic action of streptococcal enzymes, a destruction of the protective layer of heparan sulfate on the endothelium and an increase in its prothrombogenic and adhesive abilities can be seen $(3,10)$. Erovichenkov et al. (2003) demonstrated an increased level of the biomarkers for vascular wall damage, such as collagen type III,

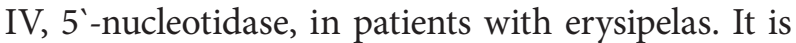
thought that the contact with collagen is an inductor of release and aggregation of cellular elements in the blood, especially the platelets. 5'- nucleotidase stimulates the transformation of prothrombin into thrombin and fibrinogen into fibrin. It is established that in patients with erysipelas, substances with thromboplastic effect - damaged endothelial and epithelial cells, erythrocytes, platelets and leucocytes - enter the blood circulation from the local site of inflammation (6).

The activation of hemostasis in patients with erysipelas is combined depending on the source of thromboplastin (3). Due to the massive release of proinflammatory mediators, as a response to the inflammation, "cytokine storm" occurs. It leads to a number of immune reactions (8). 
Radkevich (1973) first observed and described changes in hemostasis in erysipelas. His observation showed hypercoagulability running with sufficiently increased level of fibrinogen, prothrombin time, and decreased fibrinolytic activity $(3,6)$. In 1976, Dikov et al., investigated for the first time the changes in Bulgarian patients. Their results presented hyperfibrinogenemia and prolongation of thrombin time. Russian authors proposed that hemocoagulation changes were related to the development of a chronic thrombo-hemorrhagic syndrome, diminishing of blood clotting time, increase in the concentration of fibrinogen, lowering the level of fibrinogen and antithrombin 3, cessation of Hageman-dependent and induced streptokinase fibrinolysis (3). Studies of the cellular part of coagulation were conducted by Tcherkasov (1986). Other Russian authors shared their data consisting of reduced level of platelets and platelets factor IV, activation of procoagulant parts of hemostasis - increased level of fibrinogen, shortened aPTT, and increased fibrinolytic activity. The process of activation of fibrinolysis is strongly activated in bullous-hemorrhagic erysipelas. The presence of DIC in the acute phase of inflammation depends on the severity of infection and on the condition of coagulation - hypo-/hypercoagulation. In primary fibrinolysis, the following changes are observed - increased plasmin activity, reduced level of plasminogen, normal level of platelets, and increased fibrindegrading products (6).

\section{RESULTS}

Activation of the coagulation system depends on the source of thromboplastin. Thus, the mechanism of activation is often combined. Massive release of proinflammatory mediators is related to the socalled "cytokine storm", enabling the simultaneous activation of both immune and coagulation systems. In erysipelas, hemostatic changes manifest with hypercoagulability in the acute phase of disease (significantly increased level of fibrinogen, prothrombin time, reduced fibrinolytic activity). These changes are probably associated with a thrombo-hemorrhagic syndrome, shortening of the blood clotting time, increased concentration of fibrinogen, reduced level of antithrombin 3, cessation of Hageman-dependent and induced streptokinase fibrinolysis. There is a reduction in the level of platelets and the platelet factor

\section{CONCLUSION}

Coagulopathies are common findings in the pathogenesis of infectious diseases. They might be manifested as a clinical syndrome of the disease or as its complication. The spectrum of clinical findings in coagulopathies is broad enough and may vary from microthrombosis to development of DIC - syndrome, hemorrhages, hemolytic-uremic syndrome or vasculitis. As there is an upward trend in the number of patients with hemorrhagic and bullous-hemorrhagic erysipelas, as well as the presence of local and general predisposing factors, accompanying diseases and/or immunomodulating and biological therapy, there arises the need to intensify the studies in hemostasis of erysipelas in order to complete the diagnostic-therapeutic panel and prevent serious and irrevocable complications.

In patients with streptococcal infections, the presence of coagulopathy is an important clinical finding. Determining the hemostatic deviations in erysipelas and the mechanisms of their induction allows for planning in the direction of immune system modeling through the development of target therapy. Coagulopathy in erysipelas is closely connected to the inflammation response of etiological agent. Therefore, it is supposed that drugs affecting the immune system may change the direction of coagulation disorders. Low doses of antiplatelet drugs reduce the risk of multiorgan failure in septic patients. Blocking the activation of contact system may prevent severe complications. Use of heparin during erysipelas shortens the recovery time.

\section{REFERENCES}

1. Michael Y, Shaukat NM. Erysipelas. [Updated 2019 Dec 20]. In: StatPearls [Internet]. Treasure Island (FL): StatPearls Publishing; 2020 Jan-. Available from: https://www.ncbi.nlm.nih.gov/books/ NBK532247/.

2. Inghammar M, Rasmuussen $M$, Linder $A$. Recurrent erysipelas - risk factors and clinical presentations. BMC Infect Dis. 2014;14:270. doi: 10.1186/1471-2334-14-270.

3. Marina S, Kirov G. Erysipelas. Computer Art. 1992. pp. 46-9. (In Bulgarian).

4. Pahlman LI, Malmström E, Mörgelin M, Herwald H. M protein from Streptococcus pyogenes induces tissue factor expression and pro-coagulant activity 
in human monocytes. Microbiology. 2011; 153(Pt. 8): 2458-64. doi: 10.1099/mic.0.2006/003285-0.

5. Metzgar D, Zampolli A. The M-protein of group A streptococcus is a key virulence factor and a clinically relevant strain identification marker. Virulence. 2011; 2(5):402-12. doi: 10.4161/viru.2.5.16342.

6. Shannon $\mathrm{O}$, Herwald $\mathrm{H}$, Oehmcke S. Modulation of the coagulation system during severe streptococcal disease. Curr Top Microbiol Immunol. 2013; 368:189-205. doi: 10.1007/82_2012_283.

7. Hynes W, Sloan M. Secreted extracellular virulence factors. Streptococcus pyogenes: Basic biology to clinical manifestation. Oklahoma City (OK): University of Oklahoma Health Sciences Center; 2016. pp. 1-22.

8. Olsen RJ, Shelburne SA, Musser JM. Molecular mechanisms underlying group A streptococcal pathogenesis. Cell Microbiol. 2009; 11(1):1-12. doi: 10.1111/j.1462-5822.2008.01225.x.

9. Oehmcke S, Shannon O, Mörgelin M, Herwald H. Streptococcal $\mathrm{M}$ proteins and their role as virulence determinants. Clin Chim Acta. 2010; 411(1718):1172-80. doi: 10.1016/j.cca.2010.04.032.

10. Sun H, Ringdahl U, Homeister JW, Fay WP, Engleberg NC. Plasminogen is a critical host pathogenicity factor for group A streptococcal infection. Science. 2004; 305(5688):1283-6. doi: 10.1126/ science.1101245.

11. Antoniak $S$. The coagulation system in host defense. Res Pract Thromb Haemost. 2018; 2(3):54957. doi: 10.1002/rth2.12109.

12. Opal S. Interactions between coagulation and inflammation. Scand J Infect Dis. 2003; 35(9):545-54. doi:10.1080/00365540310015638.

13. Margetic S. Inflammation and haemostasis. Biochemia Medica. 2012; 22(1):49-62.

14. Nitzsche R, Rosenheinrich M, Kreikemeyer B, Oehmcke-Hecht S. Streptococcus pyogenes triggers activation of the human contact system by streptokinase. Infect Immun. 2015; 83(8):3035-42. doi: 10.1128/IAI.00180-15.

15. Loof TG, Deicke C, Medina E. The role of coagulation/fibrinolysis during Streptococcus pyogenes infection. Front Cell Infect Microbiol. 2014; 4:128. doi: 10.3389/fcimb.2014.00128.

16. Chong AY, Blann AD, Lip GYH. Assessment of endothelial damage and dysfunction: Observations in relation to heart failure. QJM - Mon J Assoc Phy- sicians. 2003; 96(4): 253-67. doi:10.1093/qjmed/ hcg037.

17. Periayah MH, Halim AS, Mat Saad AZ. Mechanism action of platelets and crucial blood coagulation pathways in hemostasis. Int J Hematol Oncol Stem Cell Res. 2017; 11(4): 319-27.

18. Linder A, Johansson 1 , Thulin P, Hertzén E, Mörgelin M, Christensson B, et al. Erysipelas caused by Group A Streptococcus activates the contact system and induce the release of heparin-binding protein. J Invest Dermatol. 2010; 130(5):1365-72; doi: 10.1038/jid.2009.437.

19. Inghammar $M$, Rasmuussen $M$, Linder $A$. Recurrent erysipelas - risk factors and clinical presentations. BMC Infect Dis. 2014; 14:270. doi: 10.1186/1471-2334-14-270.

20. Genev G, Lahtariev St, Stoicheva M, Komitova R, Geneva M. Symptoms and syndromes in infectious diseases. Sofia: Meditsina i fizkultura; 2007. pp. 167-168. (In Bulgarian).

21. Loof TG, Schmidt O, Herwald H, Theopold U. Coagulation systems of invertebrates and vertebrates and their roles in innate immunity: the same side of two coins? J Innate Immun. 2011; 3(1):34-40. doi: 10.1159/000321641.

22. Gale JA. Current understanding of hemostasis. Toxicol Pathol. 2011; 39(1):273-80. doi: $10.1177 / 0192623310389474$.

23. Fokina E. Disfibrinogenemia and hidden hemolysis - indicators of hemorrhagic syndrome in erysipelas. Global J Med R Microbiol Pathol. 2016; 16(1):1-5.

24. Fokina EG, Gerasimov AN. Prediction degree severity of erysipelas: Risk index of hemorrhagic form of erysipelas. J Infec Dis Treat. 2016; 2:2. doi: 10.21767/2472-1093.100026.

25. Sjöbring U, Ringdahl U, Ruggeri ZM. Induction of platelet thrombi by bacteria and antibodies. Blood. 2002; 100(13):4470-7. doi: 10.1182/ blood-2002-01-0069.

26. Oehmcke S, Mörgelin M, Malmström J, Linder A, Chew M, Thorlacius H, et al. Stimulation of blood mononuclear cells with bacterial virulence factors leads to the release of pro-coagulant and pro-inflammatory microparticles. Cell Microbiol. 2012; 14(1):107-19. doi: 10.1111/j.1462-5822.2011.01705.x.

27. Renné T. The procoagulant and proinflammatory plasma contact system. Semin Immunopathol. 2012; 34(1):31-41. doi: 10.1007/s00281-011-0288-2. 